\title{
The Protective Effect of EET on Renal I/R through NLRP3 and Pyroptosis
}

\author{
Ye Zhu' ${ }^{1}$, Ao Ding ${ }^{1}$, Hui Yang ${ }^{2}$, Bin-Huan Chen ${ }^{1}$, and Jun-Li Guo ${ }^{3, *}$ \\ ${ }^{1}$ Department of Nephrology, The Fifth Affiliated Hospital of Sun Yat-sen University, China \\ ${ }^{2}$ Department of Rheumatology, The Fifth Affiliated Hospital of Sun Yat-sen University, China \\ ${ }^{3}$ Department of Nephrology, Zhuhai People's Hospital, China
}

*Corresponding author: Jun-Li Guo, Department of Nephrology, Zhuhai People's Hospital (Zhuhai hospital affiliated with Jinan University), Zhuhai, China, Tel: 13417912514; E-mail: 17912514@163.com

Received: 07 Nov, 2019 | Accepted: 26 Nov, 2019 | Published: 30 Nov, 2019

Citation: Zhu Y, Ding A, Yang H, Chen BH, Guo JL (2019) The Protective Effect of EET on Renal I/R through NLRP3 and Pyroptosis. Int J Nephrol Kidney Fail 5(4): dx.doi.org/10.16966/2380-5498.186

Copyright: (c) 2019 Zhu Y, et al. This is an open-access article distributed under the terms of the Creative Commons Attribution License, which permits unrestricted use, distribution, and reproduction in any medium, provided the original author and source are credited.

\begin{abstract}
With the increasing morbidity and mortality of renal ischemia/reperfusion, whether epoxyeicosatrienoic acids could protect against it and the relative mechanism needs further exploration.

Aim: To investigate the effect of epoxyeicosatrienoic acids on renal ischemia/reperfusion injury.

Methods: Thirty mice were randomly divided into sham group, ischemia/reperfusion group, ischemia/reperfusion with epoxyeicosatrienoic acids group, ischemia/reperfusion with toll-like receptor 4 inhibitor group, ischemia/reperfusion with epoxyeicosatrienoic acids and toll-like receptor 4 inhibitor group. Kidney function and pathology changes were observed $24 \mathrm{~h}$ after surgery. ELISA was used to exam the level of serum interleukin$1 \beta$, tumor necrosis factor $\alpha$ and nod-like receptor 3 . The protein expression of nod-like receptor 3 , cleaved cysteinyl aspartate specific proteinase 1 , pro-interleukin-1 $\beta$, interleukin-1 $\beta$, toll-like receptor 4 and nuclear factor kappa-light-chain-enhancer of activated B cells p65 were evaluated by western blot.
\end{abstract}

Result: Compared with the sham group, the ischemia/reperfusion group showed severe renal tubular epithelial cell necrosis $(\mathrm{P}<0.05)$. BUN, $\mathrm{Scr}$ and interleukin-1 $\beta$, tumor necrosis factor $\alpha$, nod-like receptor 3 , toll-like receptor 4 , cleaved cysteinyl aspartate specific proteinase 1 were obviously higher than those in the sham group $(P<0.05)$. Furthermore, epoxyeicosatrienoic acids were alleviated in the ischemia/reperfusion-induced kidney function damage and expression of above protein $(P<0.05)$, the level of each proteins was significantly reduced after the co-administration of toll-like receptor 4 inhibitor and epoxyeicosatrienoic acids $(P<0.05)$.

Conclusion: Epoxyeicosatrienoic acids can reduce renal ischemia/reperfusion injury, and its mechanism may be related to the regulation of nod-like receptor 3 production and pyroptosis through the toll-like receptor 4 pathway.

Keywords: Renal ischemia/reperfusion injury; Epoxyeicosatrienoic acids; Nod-like receptor 3; Pyroptosis; Toll-like receptor 4

\section{Introduction}

The renal ischemia/reperfusion (I/R) injury is one of the major causes of Acute Kidney Injury (AKI), which is mostly induced by transplantation, cardiopulmonary resuscitation, shock, contrast nephropathy and other events [1-5]. Due to the poor prognosis and lack of effective therapy, AKI is markedly associated with a high morbidity and mortality [6,7]. As a vital pathophysiological process of I/R, nod-like receptor 3 (NLRP3) inflammasome acts as a trigger of the aseptic inflammation, which stimulates the laminar response of immune cells and inflammatory factors, and then results in tubular damage [8-10]. Recent findings indicated that pyroptosis is a special form of programmed cell death, which is dependent on inflammatory responses, and plays an important role in I/R injury $[11,12]$. Therefore, the inhibition of inflammation and reduction of pyroptosis may be a necessary target of preventing I/R-induced kidney injury.
Epoxyeicosatrienoic acids (EETs), metabolized from arachidonic acid through the cytochrome P450 epoxygenases pathway, have such protective effects of vasodilation, anti-inflammation and antiapoptosis in the treatment of multiple diseases [13-15]. Our previous evidence showed that EETs can alleviate inflammatory cell infiltration induced by renal I/R [16-18]. In this study, male C57/BL6 mice were used to establish I/R-induced AKI model with the pre-administration of 14,15-EET. The expression of NLRP3, cleaved cysteinyl aspartate specific proteinase 1 (cleaved-caspase-1), interleukin-1 $($ IL-1 $\beta$ ) and so on were measured to analyze the effect and mechanism of EET on renal I/R-related inflammatory response, which may be developed a novel and useful treatment to improve AKI.

\section{Materials and Methods}

The experimental protocols with mice were duly approved by 
the Institutional Animal Ethics Committee of The Fifth Affiliated Hospital of Sun Yat-sen University, 10 week old C57/BL6 mice were randomly divided into five groups ( $\mathrm{n}=6$ per group): sham group, I/R group, I/R+EET group, IR+TLR4 inhibitor (TAK-242) group, $\mathrm{IR}+\mathrm{EET}+\mathrm{TAK}-242$ group. Mice were anesthetized by isoflurane and then EET, TAK-242 were intraperitoneal injected 1 hour before $\mathrm{I} / \mathrm{R}$ operation. The temperature of the mice was maintained at $37^{\circ} \mathrm{C}$ throughout the operation. The I/R mice model was performed by bilateral renal pedicle clamping for 18 minutes and then the clamp was removed to initiate reperfusion. Mice in sham group underwent the same protocol without clamping renal pedicle. The mice were sacrificed 24 hours after the surgery, and the blood and kidney specimens were collected for further analysis.

\section{Reagent}

The 14,15-EET analogue was purchased from Cayman Chemical Company (Item:10009286). NLRP3 (\#13158), cleaved-caspase-1

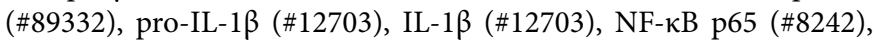
GAPDH (\#2118) and Goat anti-rabbit were purchased from CST. Tolllike receptor 4 (TLR4) (A5258) was purchased from ABclonal. ELISA kit was purchased from Wuhan BOSTER Biological Technology Co, Ltd.

\section{Animals}

Male C57BL6 mice (10-week-old) weighing 20-25 g were purchased from Nanjing University-Nanjing Institute of Biomedicine, No: SYXK (Su) 2015-0001. Regular food and tap water were given and the mice were maintained on a 12-h light/dark cycle in SPF environment of the Molecular Imaging Center of the Fifth Affiliated Hospital of Sun Yatsen University.

\section{Biochemical analysis}

The level of blood urea nitrogen (BUN) and serum creatinine (Scr) were detected by biochemical kit (Figure 1).

\section{Histological analysis of renal injury}

The kidney tissue was fixed with $10 \%$ formalin and then embedded with paraffin, placed on slides, stained with Hematoxylin and Eosin $(\mathrm{H} \& \mathrm{E})$, the staining sections were observed using a microscope.

\section{ELISA}

The serum levels of IL-1 $\beta$, TNF- $\alpha$ and NLRP3 were examined by ELISA kits according to the manufacturer's instructions.

\section{Western blot analysis}

Kidney tissue extraction and the protein concentration detection was according to the BCA protein assay kit (Beyotime, China, P0010). Samples $(30 \mu \mathrm{g}$ per lane) were separated by $15 \%$ SDS-PAGE, $10 \%$ SDS-PAGE and transferred into Polyvinylidene difluoride (PVDF) membranes. After blocking the membranes with 5\% non-fat milk for 1 hour at room temperature, the membranes were applied with the primary antibodies of rabbit polyclonal antibody against NLRP3 (1:1000), cleaved-caspase-1(1:1000), pro-IL-1 $\beta$ (1:1000), IL-1 $\beta$ (1:1000), NF-kB p65 (1:1000), TLR4 (1:1000) and GADPH (1:1000) at $4^{\circ} \mathrm{C}$ overnight, and then incubated with goat anti-rabbit for 1 hour at room temperature. ECL chemiluminescence was used to detect protein expression, and band density was quantified by Image J software.

\section{Statistical analyses}

Experimental data were represented as means \pm standard error of mean (SEM) and analyzed by SPSS 22.0. Differences in multiple groups were analyzed using one-way ANOVA. Differences with $\mathrm{P}<0.05$ were considered as statistically significant.

\section{Result}

\section{EET reduces $I / R$-induced kidney function damage}

To evaluate the effect of EET in I/R injury, BUN and Scr levels were significantly up-regulated in $\mathrm{I} / \mathrm{R}$ group than those in the sham group $(\mathrm{P}<0.05)$. With the EET pre-administration, BUN and Scr were notably decreased compared to I/R group $(\mathrm{P}<0.05)$.

\section{EET extenuates the I/R-induced tubular necrosis}

Normal glomeruli, renal tubules and interstitial architecture were observed in the sham group (Figure 2). The detachment of brush border and some renal tubular epithelial cells necrosis were observed in the $\mathrm{I} / \mathrm{R}$ group $(\mathrm{P}<0.05)$. However, the renal tubular injury is not obvious with EET treatment after I/R surgery. Treatment of EET improves the renal damage than those in $I / R$ group $(\mathrm{P}<0.05)$.
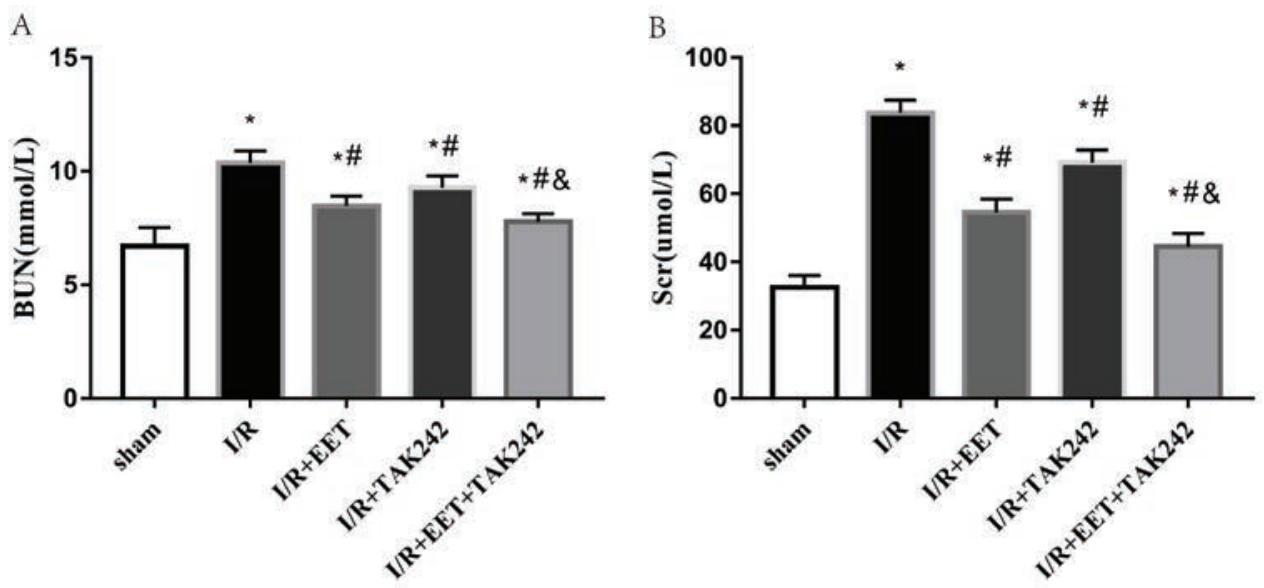

Figure 1: BUN and Scr were measured in mice

*P $<0.05$ vs sham group; \#P $<0.05$ vs I/R group; \&P $<0.05$ vs $\mathrm{I} / \mathrm{R}+\mathrm{EET}$ group. Mean $\pm \mathrm{SD} . \mathrm{N}=6$ per group. 


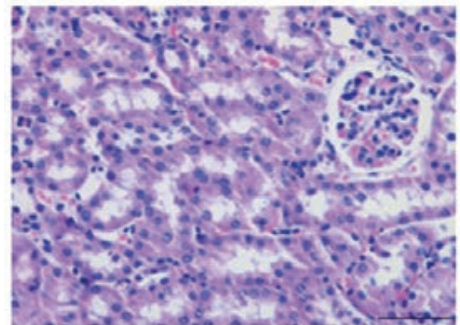

A.sham

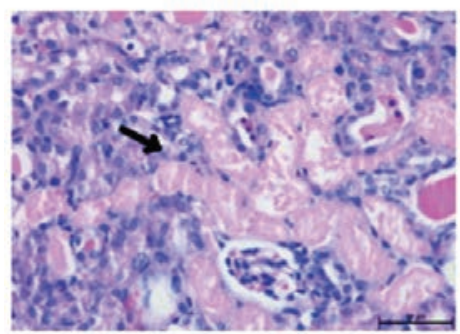

D.I/R+TAK 242

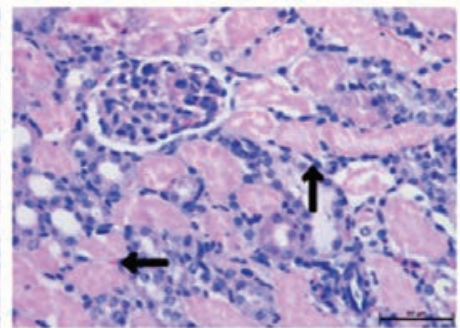

B.I/R

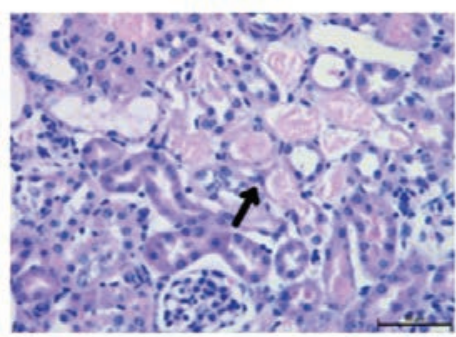

E.I/R+EET+TAK 242

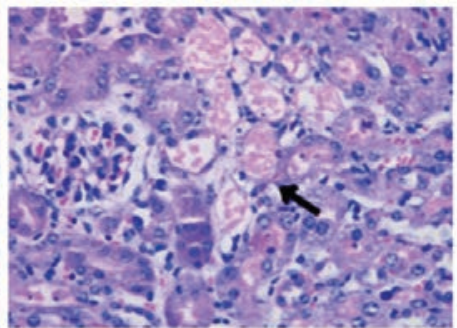

C.IR+EET

Figure 2: Representative histology of renal tissue is deserved in different group (HE staining, $\times 400)$.

A: sham group; B: ischemia/reperfusion (I/R) group; C: I/R group with EET; D: I/R group with TAK242; E: I/R group with EET and TAK242.

Mean \pm SD. $\mathrm{N}=6$ per group.

A

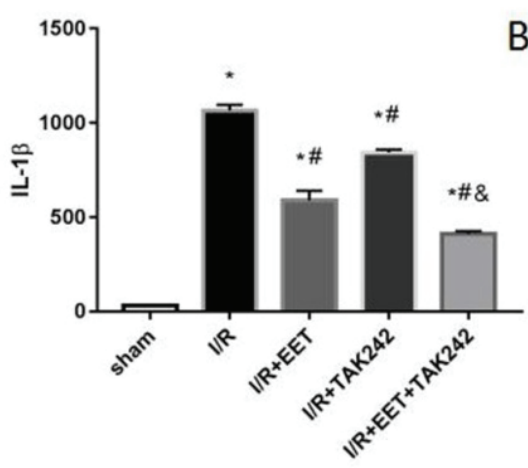

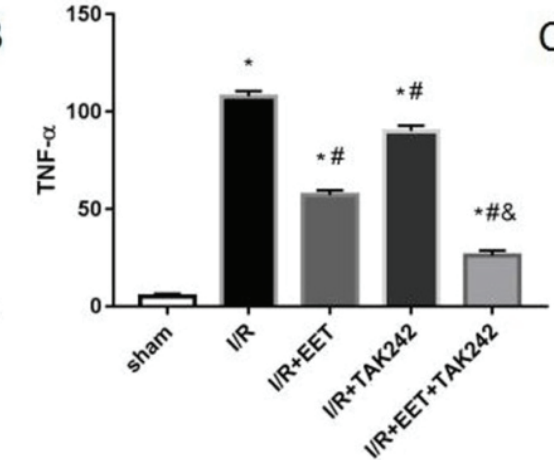

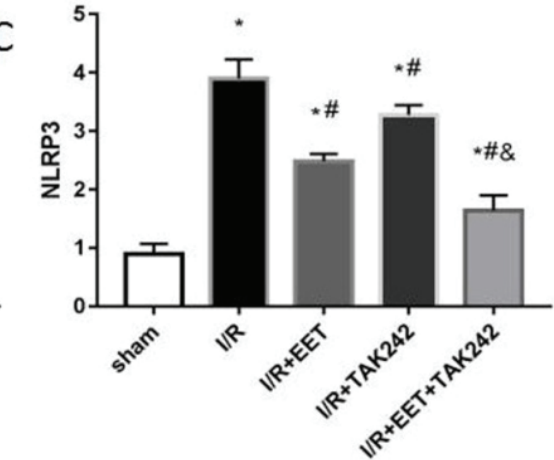

Figure 3: The serum level of IL-1 $\beta$, TNF- $\alpha$, NLRP3 in mice.

${ }^{*} \mathrm{P}<0.05$ vs. sham group; $\# \mathrm{P}<0.05$ vs. I/R group; $\& \mathrm{P}<0.05$ vs. I/R+EET group.

Mean $\pm \mathrm{SD}$. $\mathrm{N}=6$ per group.

\section{EET decreases the serum levels of inflammatory factors and NLRP3}

To observe the inflammatory changes, ELISA kit was used to detect the levels of serum inflammatory factors to access the effect of EET on renal inflammation (Figure 3 ). The levels of serum IL-1 $\beta$, TNF- $\alpha$ and NLRP3 in the I/R group were significantly up-regulated in comparison to the Sham group $(\mathrm{P}<0.05)$. While the levels were decreased after being treated with EET than those in $I / R$ group $(\mathrm{P}<0.05)$.

\section{EET may regulate NLRP3 expression and pyroptosis by TLR4 pathway}

The expression of inflammasome and pyroptosis-related NLRP3, cleaved-caspase-1, pro-IL-1 $\beta$, IL-1 $\beta$, TLR4 were evaluated by western blot (Figure 4). Compared with the sham group, there was an obviously increase of NLRP3, cleaved-caspase-1, pro-IL-1 $\beta$, IL-1 $\beta$ in I/R group $(\mathrm{P}<0.05)$, while EET appeared to decrease the expression of the above proteins $(\mathrm{P}<0.05)$. In addition, to further investigate the mechanism of EET result to the activation of NLRP3 and pyroptosis, we detected the expression of TLR4 and NF- $\kappa B$ p65 after I/R surgery with EET and/ or TLR4 inhibitor (TAK-242). The result indicated that EET mitigated above protein expression, while the co-administration of EET and TAK-242 enhanced the inhibitory effect of EET alone on NLRP3 and pyroptosis $(\mathrm{P}<0.05)$.

\section{Conclusion}

As a severe disease with high morbidity in China, AKI brings 
a huge physical and economic burden to patients. Furthermore, the pathophysiological mechanism of AKI is complicated and it could be caused by multiple factors, including surgery, hypoxia and inflammation. In the process of renal ischemia and hypoxia, injured vascular endothelial cells stimulate the cascade of inflammation, which lead to tubular and interstitial damage. Previous evidence revealed that inflammasome is the critical core in inflammation about mediating cell death, among them, NLRP3 inflammasome as a key factor in this inflammation cascade, by self-oligomerization and signal transduction, causing renal injury. After inhibiting the occurrence of inflammasome, it can regulate the maturation and release of pro-inflammatory cytokines and reduce the kidney damage. In the current study, I/R group notably showed that the increase of BUN, Scr and tubular damage, detachment of brush border. However, when treated with EET, there was a decline in BUN, Scr, NLRP3, IL- $1 \beta$ and TLR4, suggesting that EET alleviate I/Rinduced inflammation and renal dysfunction.
Pyroptosis, which differ from necrosis and apoptosis, is a special inflammatory-way of programmed cell death. The activation of cleaved-caspase-1, as a vital factor promotes the maturation of IL$1 \beta$ and other inflammatory cytokines under the stimulation of an endogenous and exogenous effect accompanied by the procession of an inflammation cascade and cell death. Additionally, the pathway of TLR4 and NLRP3 inflammasome can be activated under the trigger of exogenous pathogen-associated molecular patterns (PAMPs) and endogenous Danger-Associated Molecular Patterns (DAMPs), thus also mediating the inflammation response and pyroptosis. NLRP3 as a regulator in the cleavage of pro-IL- $1 \beta$, activated TLR4 pathway induces the transcription of pro-IL-1 $\beta$. Accordingly, there may be a collaborating effect between TLR4 pathway and NLRP3 inflammasome in activating IL- $1 \beta$. Simultaneously, there may be also a strong link between inflammasome and pyroptosis. Recent studies showed that pyroptosis is the key in kidney disease by
A

Cleaved-caspase-1

Pro-IL-1 $\beta$

IL-1 $\beta$

TLR4

NF-kB P65

GAPDH

I/R

EET

TLR4

B

C

E
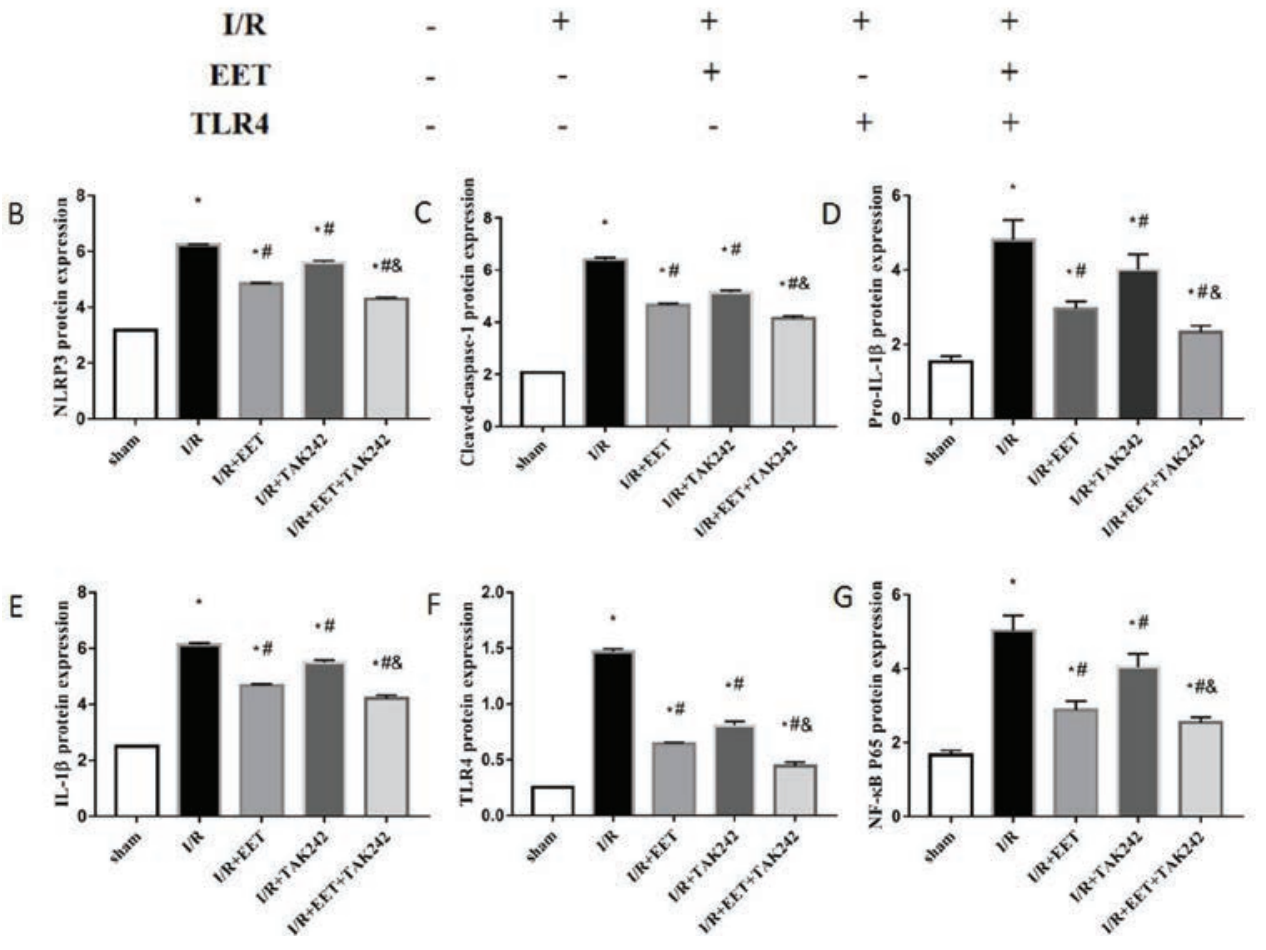

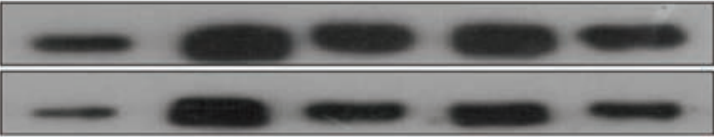

$110 \mathrm{kDa}$

\section{2kDa}

$31 \mathrm{kDa}$

$17 \mathrm{kDa}$

$120 \mathrm{kDa}$

$65 \mathrm{kDa}$

37kDa

Figure 4: Immunoblots and quantitation of NLRP3, cleaved-caspase-1, pro-IL-1 $\beta$, IL-1 $\beta$, TLR4, NF-KB P65 protein expression. Mean \pm SD. $\mathrm{N}=6$ per group.

*P<0.05 vs. sham group; \#P<0.05 vs. I/R group; \&P<0.05 vs. I/R+EET group. 
NLRP3-caspase-1 pathway. The mice were designed to induce I/R model in this study. The levels of NLRP3, cleaved-caspase-1, IL- $1 \beta$ were markedly increased in the $\mathrm{I} / \mathrm{R}$ group than the sham group. While NLRP3, cleaved-caspase-1, IL-1 $\beta$ were decreased after the application of EET, which determined the inhibition of NLRP3 inflammasomes activation because of the EET treatment, confirmed by western blot, showed that EET resulted in a marked reduction of inflammationrelated protein levels and mitigated the formation of inflammasome as well as pyroptosis.

TLR4 pathway is a classical pathway in activating NLRP3 inflammasome. Activated TLR4 finally activates NF- $\kappa B$ by complicated cascade reactions to mediate other inflammation and immune responses. The activation of the TLR4 pathway induces the transcription of pro-IL-1 $\beta$, while the activation of NLRP3 inflammasome induces the cleavage of IL-1 $\beta$. In summary, there is a collaborating effect between the TLR4 pathway and NLRP 3 inflammasome in activating IL-1 $\beta$. Meanwhile, the release of inflammatory injury factors caused by pyroptosis can continue to serve as an endogenous ligand for TLR4, forming a positive feedback to aggravate inflammatory damage. Considering the relationship and mechanism of the TLR4 pathway, NLRP3 inflammasome and pyroptosis, I/R was surgically induced in mice with EET or/and TAK-242 administration in this experiment. The data revealed that EET decreased the levels of BUN, Scr, NLRP3, TNF- $\alpha$, TLR4, cleaved-caspase- 1 and IL- $1 \beta$, exerting a beneficial impact in extenuating inflammation response and pyroptosis. While the co-administration of EET and TAK242 reduced the above levels even more, as well as enhancing the useful effect, indicating that the TLR4 pathway was activated in renal I/R injury and probably plays a vital role with NLRP3 inflammasome in I/R-induced inflammation and pyroptosis pathway. It could be conclusion that EET may provide a protective role in renal I/R. The exact pharmacological mechanism of it maybe associates with NLRP3 and pyroptosis.

\section{Grants}

This study was supported in part by grants of the National Natural Science Foundation of China (NSFC No.81600529), the Fundamental Research Funds of the Central Universities (17ykpy64), Science and Technology Program of Zhuhai, China (20181117E030069).

\section{References}

1. Aiyegbusi O, Witham MD, Lim M, Gauld G, Bell S (2019) Impact of introducing electronic acute kidney injury alerts in primary care. Clin Kidney J 12: 253-257.

2. Borasino S, Kalra Y, Elam AR, Carlisle O'Meara L, Timpa JG, et al. (2018) Impact of Hemolysis on Acute Kidney Injury and Mortality in Children Supported with Cardiac Extracorporeal Membrane Oxygenation. J Extra Corpor Technol 50: 217-224.

3. Cima L, Nacchia F, Ghimenton C, Valotto G, Boschiero L, et al. (2019) Histopathology and Long-Term Outcome of Kidneys Transplanted from Donors with Severe Acute Kidney Injury. Prog Transplant 29: 36-42.
4. Di Bacco L, Montisci A, Miceli A (2019) Commentary: Acute kidney injury: The one-million-pieces puzzle. J Thorac Cardiovasc Surg 157: 2367-2368.

5. Eun Ju Lee, Ha Nee Jang, Hyun Seop Cho, Eunjin Bae, Tae Won Lee, et al. (2018) The incidence, risk factors, and clinical outcomes of acute kidney injury (staged using the RIFLE classification) associated with intravenous acyclovir administration. Ren Fail 40: 688-693.

6. Chatterjee S, LeMaire SA, Amarasekara HS, Green SY, Price MD, et al. (2019) Early-Stage Acute Kidney Injury Adversely Affects Thoracoabdominal Aortic Aneurysm Repair Outcomes. Ann Thorac Surg 107: 1720-1726.

7. Mohammadi Kebar S, Hosseini Nia S, Maleki N, Sharghi A, Sheshgelani A (2018) The Incidence Rate, Risk Factors and Clinical Outcome of Acute Kidney Injury in Critical Patients. Iran J Public Health 47: 1717-1724.

8. Lei Q, Yi T, Chen C (2018) NF-KB-Gasdermin D (GSDMD) Axis Couples Oxidative Stress and NACHT, LRR and PYD Domains-Containing Protein 3 (NLRP3) Inflammasome-Mediated Cardiomyocyte Pyroptosis following Myocardial Infarction. Med Sci Monit 24: 6044-6052.

9. Li Q, Ye T, Long T, Peng X (2019) Ginkgetin exerts anti-inflammatory effects on cerebral ischemia/reperfusion-induced injury in a rat model via the TLR4/NF-KB signaling pathway. Biosci Biotechnol Biochem 83: 675-683.

10. Wang S, Yuan YH, Chen NH, Wang HB (2019) The mechanisms of NLRP3 inflammasome/pyroptosis activation and their role in Parkinson's disease. Int Immunopharmacol 67: 458-464.

11. Dong Z, Pan K, Pan J, Peng Q, Wang Y (2018) The Possibility and Molecular Mechanisms of Cell Pyroptosis after Cerebral Ischemia. Neurosci Bull 34: 1131-1136.

12. Toldo S, Mauro AG, Cutter Z, Abbate A (2018) Inflammasome, pyroptosis, and cytokines in myocardial ischemia-reperfusion injury. Am J Physiol Heart Circ Physiol 315: H1553-H1568.

13. Guo Y, Luo F, Zhang X, Chen J, Shen L, et al. (2018) TPPU enhanced exercise-induced epoxyeicosatrienoic acid concentrations to exert cardioprotection in mice after myocardial infarction. J Cell Mol Med 22: $1489-1500$.

14. Hye Khan MA, Fish B, Wahl G, Sharma A, Falck JR, et al. (2016) Epoxyeicosatrienoic acid analogue mitigates kidney injury in a rat model of radiation nephropathy. Clin Sci (Lond) 130: 587-599.

15. Imig JD (2016) Epoxyeicosatrienoic Acids and 20-Hydroxyeicosatetraenoic Acid on Endothelial and Vascular Function. Adv Pharmacol 77: 105-141.

16. Hoff U, Bubalo G, Fechner M, Blum M, Zhu Y, et al. (2019) A synthetic epoxyeicosatrienoic acid analogue prevents the initiation of ischemic acute kidney injury. Acta Physiol (Oxf) 227: e13297.

17. Khan MA, Liu J, Kumar G, Skapek SX, Falck JR, et al. (2013) Novel orally active epoxyeicosatrienoic acid (EET) analogs attenuate cisplatin nephrotoxicity. FASEB J 27: 2946-2956.

18. Romashko M, Schragenheim J, Abraham NG, McClung JA (2016) Epoxyeicosatrienoic Acid as Therapy for Diabetic and Ischemic Cardiomyopathy. Trends Pharmacol Sci 37: 945-962. 